PITHA - 96/38

\title{
Edge States and Entropy of 2d Black Holes
}

\author{
J. Gegenberg $\dagger$ G. Kunstatter $\sharp$ T. Strobl $b$ \\ $\dagger$ Dept. of Mathematics and Statistics, University of New Brunswick \\ Fredericton, New Brunswick, Canada E3B 5A3 \\ [e-mail: lenin@math.unb.ca] \\ $\sharp$ Dept. of Physics and Winnipeg Institute of Theoretical Physics, \\ University of Winnipeg \\ Winnipeg, Manitoba, Canada R3B 2E9 \\ [e-mail: gabor@theory.uwinnipeg.ca] \\ b Institut für Theoretische Physik, RWTH-Aachen \\ Sommerfeldstr. 26-28, D52056 Aachen, Germany \\ [e-mail: tstrobl@physik.rwth-aachen.de]
}

\begin{abstract}
In several recent publications Carlip, as well as Balachandran, Chandar and Momen, have proposed a statistical mechanical interpretation for black hole entropy in terms of "would be gauge" degrees of freedom that become dynamical on the boundary to spacetime. After critically discussing several routes for deriving a boundary action, we examine their hypothesis in the context of generic 2-D dilaton gravity. We first calculate the corresponding statistical mechanical entropy of black holes in $1+1$ deSitter gravity, which has a gauge theory formulation as a BF-theory. Then we generalize the method to dilaton gravity theories that do not have a (standard) gauge theory formulation. This is facilitated greatly by the Poisson $\sigma$-model formulation of these theories. It turns out that the phase space of the boundary particles coincides precisely with a symplectic leaf of the Poisson manifold that enters as target space of the $\sigma$-model. Despite this qualitatively appealing picture, the quantitative results are discouraging: In most of the cases the symplectic leaves are non-compact and the number of microstates yields a meaningless infinity. In those cases where the particle phase space is compact - such as, e.g., in the Euclidean deSitter theory - the edge state degeneracy is finite, but generically it is far too small to account for the semiclassical Bekenstein-Hawking entropy.
\end{abstract}




\section{Introduction}

The idea that black holes have entropy [1] has presented theoretical physics with one of its most important and puzzling challenges in recent years. If it is true that we are to assign entropy to a black hole, then according to standard wisdom of thermodynamics, there should also be a microscopic, statistical mechanical explanation for this entropy. There has been considerable progress in this direction along two very different fronts. Strominger and Vafa [2] have been able to derive the Bekenstein-Hawking entropy by counting quantum mechanical string states for the case of static, five-dimensional extreme black holes. Their results have also been extended to a large variety of extreme and near extremal black holes (see [3] for a recent review). In all these cases, the degeneracy originates in the newly discovered nonperturbative symmetries in the BPS spectra of string and brane theory.

An alternative and in principle more general attempt to explain black hole entropy has been developed by Carlip [由, 5] as well as by Balachandran, Chandar, and Momen [6]. The main idea is roughly as follows: Classically, one is unable to find out what is happening behind the horizon of a black hole. Correspondingly, when quantizing, one should restrict consideration to the part of spacetime exterior to the horizon (or 'stretched horizon' [7]). This leads to the investigation of gravitational systems on a manifold with boundary.] Given a Lagrangian system with gauge symmetries on a manifold with boundary, Carlip as well as Balachandran et al. argue that an appropriate incorporation of boundary conditions leads to physical observables that would be absent in the case of a manifold without boundary. These 'additional' degrees of freedom may be thought of as living on the boundary, a mechanism that is also utilized in a theoretical description of the quantum Hall effect [11, 6]. After quantization, these modes should give rise to edge states which can in principle be traced out in order to account for the black hole entropy.

The existence of boundary degrees of freedom can be seen most easily in a Hamiltonian formulation [6]: Gauge symmetries give rise to (first class) constraints $G^{i}(x) \approx 0$ in the phase space (here the $x$ are coordinates on the

\footnotetext{
${ }^{1}$ There are also further possible motivations for considering a manifold with boundary, cf. [4, 6]. One quite different alternative, advocated by T. Jacobson [8] as well as the school of York [9], is to think of the boundary as an outer boundary leading to a 'gravitational system in a box'. Another related approach is that of Maggiore, in which the microstates are associated with strings propogating in regions of spacetime near the event horizon [10].
} 
spatial part $\Sigma$ of our manifold $\mathcal{M}=\Sigma \times \mathbb{R}$ ). More correctly the $G^{i}$ should be smeared by some test functions (the Lagrange multiplier fields):

$$
\mathcal{G}_{\epsilon}:=\int \epsilon_{i}(x) G^{i}(x) d x \approx 0
$$

Generically the $G^{i}$ will include some spatial derivatives acting on canonical variables. Suppose, for definiteness, that the $G^{i}$ 's are of the form

$$
G^{i}=\partial X^{i}(x)+F^{i}(X(x), \ldots)
$$

where $X^{i}(x)$ denote some canonical variables and $F^{i}$ are ordinary functions of $X^{i}(x)$ and possibly some further canonical variables. To ensure functional differentiability of the constraints $\mathcal{G}_{\epsilon}$, i.e. to ensure well-defined Hamiltonian vector-fields corresponding to (1), the functions $\epsilon_{i}(x)$ are required to vanish at the spatial boundary $\partial \Sigma$. Thus constraints are those functions (11) on the phase space for which $\left.\left(\epsilon_{i}\right)\right|_{\partial \Sigma}=0$. Under these assumptions, phase space function(al)s

$$
O_{\xi}:=-\int\left[X^{i}(x) \partial \xi_{i}(x)-F^{i}(X(x), \ldots) \xi_{i}(x)\right] d x
$$

with $\left.\left(\xi_{i}\right)\right|_{\partial \Sigma} \neq 0$ do not necessarily vanish on-shell. They coincide with the constraints only up to boundary contributions on $\partial \Sigma$. Nonetheless, they give rise to a well-defined Hamiltonian vector field. Moreover, their Poisson brackets with the constraints (四) will reflect the constraint algebra; the only difference will be the kind of test functions appearing at the r.h.s. of the brackets. It then often will happen - and in the case of four-dimensional gravity and the models considered below it in fact does happen - that the vanishing of $\epsilon_{i}$ on the boundary is sufficient to guarantee that $\left\{O_{\xi}, \mathcal{G}_{\epsilon}\right\} \approx 0$. If, furthermore, the boundary conditions imposed on the fields do not fix $O_{\xi}$ completely, Eqs. (3) give rise to non-trivial gauge invariant (i.e. physical) observables, associated to the spatial boundary $\partial \Sigma$. (Cf. [6] for more details).

It may, however, be difficult to locate explicitly all the boundary degrees of freedom obtained in the above manner, particularly when the "gauge group" is actually the group of spacetime diffeomorphisms. It is for this reason that Carlip examined the simplified model of $2+1$ Einstein gravity with cosmological constant. This theory admits black hole solutions discovered by Banados, Teitelboim and Zanelli (BTZ) [12]. Moreover, it has a gauge theoretical formulation as a Chern-Simons theory with gauge group $\mathrm{SO}(2,2)$ [13]. Carlip 
showed that if the spacetime $\mathcal{M}$ has a boundary $\partial \mathcal{M}$, a surface term must be added to the usual Chern-Simons action functional in order to make the variational principle well defined. For boundary conditions required so as to make $\partial \mathcal{M}$ an event horizon, the surface term is of the form of a WessZumino-Novikov-Witten (WZNW) $\sigma$-model coupled to external currents and the entropy obtained by quantizing the boundary modes turns out to indeed coincide (to leading order) with the semiclassical 2+1 Bekenstein-Hawking entropy [4, 5]. Unfortunately, the quantization is not well-understood for non-compact gauge groups like $S O(2,2)$, except in the so-called infinite coupling limit. In his original calculation, Carlip was able to use the fact that in this limit the WZNW theory reduces to a bosonic string theory, in which the degeneracy of the microstates is calculable. In a more recent calculation Carlip has applied this program to the Euclidean black hole in $2+1$ gravity [5]. In this case the gauge group is $\operatorname{SL}(2, \mathrm{C})$. Although this is still non-compact, Carlip makes use of a result due to Witten [14] which relates the partition function for the SL $(2, \mathrm{C})$ theory to the product of partition functions for a corresponding SU(2) Chern-Simons theory. The quantum mechanics in the latter case is much better understood and Carlip was able to derive a result that agrees with the one obtained in the Lorentzian case.

Attempts to generalize this calculation to the more realistic setting of $3+1$ gravity have failed thus far. The main reason for this is that the $2+1$ analysis relies very heavily on the gauge theoretic formulation of the theory, which does not exist for $3+1$ gravity. In the present paper we intend to study the above statistical mechanical approach to the entropy of black holes within an even lower dimensional context, namely $1+1$ dimensional gravity. There are at least two good reasons for doing so: First of all, it will bring about considerable technical simplification while still allowing for a conceptually similar setting. Since the boundary of a two-dimensional spacetime is one-dimensional, the edge degrees of freedom will be purely mechanical ones. Consequently the (coupled) WZNW model in Carlip's calculation is replaced by a point particle model with a finite dimensional phase space. The mathematics involved is therefore more straightforward, allowing one to focus on conceptual rather than technical issues.

The second motivation for considering 2-D gravity concerns the universal validity of Carlip's approach. As Wald's work emphasizes [15], the classical thermodynamical behaviour associated with event horizons can be derived in a model-independent way. Only the details differ from one theory to another. This raises the following important question: Do edge states account for 
entropy in any geometrical theory that admits solutions with event horizons? Although we will not be able to answer this question definitively, we will be able to apply Carlip's method to a large class of 2-D gravity theories. In this context it is particularly important to note that almost all of the 2-D models considered below do not allow for a formulation in terms of an ordinary gauge theory. We are nonetheless able to generalize and apply the methods developed for theories such as $2+1$ gravity that do have a gauge theoretic formulation. These models therefore provide the first examples of non-gauge theoretic models where the approach of Carlip and Balachandran et al. may be applied and tested.

Somewhat surprisingly, we do not get the expected results for any of the models considered. It is not clear at this stage whether this is due to the special features (i.e. finite number of degrees of freedom) of the edge dynamics in 2-D, or whether it indicates that the general method lacks universal validity in its present form.

The theories we wish to consider include the general class of dilaton gravity models governed by a Lagrangian for a 2 d metric $g$ and a dilaton field $\Phi$ of the form [16]

$$
L_{\mathrm{gdil}}[g, \Phi]=\int_{M} d^{2} x \sqrt{|\operatorname{det} g|}\left[D(\Phi) R-V(\Phi)+Z(\Phi) g^{\mu \nu} \partial_{\mu} \Phi \partial_{\nu} \Phi\right] .
$$

Our considerations will be applicable to any Lagrangian of the above form (provided that the functions $D, V, Z$ are smooth, $D^{\prime} \neq 0$ and either $Z \neq 0$ or $Z \equiv 0)$. However, for simplicity we will mostly restrict ourselves to the case $D=\Phi$ and $Z \equiv 0.2$ The deSitter model results from (41) by further choosing $V \propto \Phi$. Only in this particular case, as well as for $V=$ const (reformulated string inspired gravity), the theory (雨) (with $D=\Phi$ and $Z \equiv 0$ ) may be reformulated entirely in terms of a standard gauge theory [18, 19]. For other choices of the 'potential' $V$ we have to leave the realm of ordinary gauge theories, and, consequently, from this perspective we are closer in spirit to the $4 \mathrm{~d}$ theory than in Carlip's $2+1$ treatment. It should also be noted that (4) incorporates the spherical reduction of the Einstein Hilbert action [20]. Thus our treatment should also cover the four-dimensional Schwarzschild black hole.f Let us remark, however, that in the present context the suppressed

\footnotetext{
${ }^{2}$ Actually this is not much of a restriction: Under the above assumptions this form of $L_{\text {gdil }}$ may be obtained always by a redefinition of the basic fields (e.g., $\Phi \rightarrow D(\Phi)$ ) 17 .

${ }^{3}$ With a slight generalization of (酒) we may also treat charged black holes. Only for rotating black holes other techniques would be required.
} 
dimensions could become vital; the handling of the boundary may break the rotational invariance (or additional fields may be needed to restore it). Finally, we note that using the general considerations of Wald [15], or a straightforward analysis specific to the present class of models [21], it can be shown that for solutions to (田) with event horizons, the thermodynamical entropy is generically given by

$$
S_{\text {thermo }}=\frac{4 \pi D\left(\Phi_{0}\right)}{\hbar}
$$

where $\Phi_{0}$ is the value of the dilaton field at the outer horizon. At the end of Section 2 one of several derivations of (5) will be recapitulated briefly.

The specific model closest in spirit to Carlip's original calculation is that of deSitter gravity, for which $V \propto \Phi$. This theory can be written in gauge theoretic form as a BF topological field theory [18] with Lie algebra so(2,1). Moreover, it can be obtained by dimensional reduction (imposing axial symmetry) on $2+1$ gravity. Thus, the black hole solutions in this model are the dimensionally reduced versions of the BTZ black hole [22]. We will show that the boundary action of the deSitter model turns out to be that of a coadjoint orbit. The quantization of such a system is standard and straightforward. We provide a brief review of various methods for doing this in an Appendix, including a method recently developed [23] for the case of Euclidean black holes in 2-D deSitter gravity, in which the system can be quantized in terms of a system of two harmonic oscillators with fixed total energy. Quantization in this case yields a discrete mass spectrum and the degeneracy of states turns out to be proportional to the square root of the mass. The resulting entropy of $\ln \sqrt{M}$ disagrees with the Bekenstein-Hawking value, which is proportional to $\sqrt{M}$.

As previously noted in the case of general 2-D dilaton gravity a gauge theoretic formulation does not exist. The main tool for treating the general class of models (俩) will thus be their equivalent formulation as so-called Poisson $\sigma$-models [25] (cf. also [26, 27]). From a certain perspective the latter are non-linear gauge theories [28, 29]. For us it will be more important, however, to regard them as $\sigma$-models of a very special kind: While spacetime is the worldsheet of the model, its target space is a 'Poisson manifold'. This means that the target space of the theory, which is just $\mathbb{R}^{3}$ in the

\footnotetext{
${ }^{4}$ We note, however, that the logarithmic result is in qualitative agreement with a numerical investigation of the entanglement entropy of $1+1$ dimensional gravity systems performed by Srednicki 24.
} 
present context, carries a Poisson bracket. This Poisson bracket is degenerate. On restriction to appropriate two-dimensional submanifolds (of the

$\mathbb{R}^{3}$ ), the bracket becomes non-degenerate; thus these submanifolds are symplectic and the full three-dimensional target space foliates into (generically) two-dimensional symplectic leaves. It turns out that a target space coordinate (i.e. a certain function of the fields of the $\sigma$-model), which may be used to label the different symplectic leaves, may be identified on-shell with the mass $M$ of the black holes of the classical solutions.

The knowledge of this somewhat unexpected structure has been used in several works to efficiently solve the general models (4) at both the classical and quantum level [25]. In the present context, this hidden Poisson structure will become even more important. On our (spacetime) manifold with boundary we will fix boundary conditions so as to pick out a black hole of fixed mass $M$. We will then derive the point particle action induced at the boundary following the strategy of [1, 6]. The phase space of this point particle will turn out to be identical to the symplectic leaf singled out by the choice of $M$ ! So, whereas in previous works the Poisson structure was used for auxiliary purposes in solving (14), now the symplectic leaves of the target space become 'alive': they provide the phase space for the edge dynamics. Thus, the final picture that emerges for general 2-D dilaton gravity is very appealing. Still, it does not seem to yield reasonable results for the statistical mechanical entropy.

The paper is organized as follows: In Section 2 we review the logic of [4, 6] for obtaining the WZNW action governing the boundary degrees of freedom in the $2+1$ theory. In the process, we point out some puzzling conceptual features of the approach, which are then made even more explicit when applying the procedure to $2 \mathrm{~d}$ deSitter gravity. In Section 3 the discussion is extended to the case of general 2-D dilaton gravity. Section 4 concludes with a short summary and outlook.

\section{Gauge-Theory-like Gravity Models}

As shown in [13], 2+1 gravity, with or without a cosmological constant $\Lambda$, may be formulated in terms of a Chern-Simons gauge theory

$$
C S[A]=\frac{k}{4 \pi} \int_{\mathcal{M}} \operatorname{tr}\left(A \wedge d A+\frac{2}{3} A \wedge A \wedge A\right)
$$


Here $A$ is a standard gauge field, i.e. a Lie-algebra valued one-form. For $\Lambda \neq 0$ the appropriate gauge group is $S O(2,1) \times S O(2,1)$ for Lorentzian signature and $S L(2, C)$ for the Euclidean signature of the gravity theory. So $A$ is a collection of six one-forms, three of which may be identified with the threebein of the gravity theory, while the remaining ones coincide with the three components of the spin-connection.

As required for a gravity theory, (6) is invariant under diffeomorphisms. If $\mathcal{M}$ has no boundary, (6) is also invariant with respect to non-abelian gauge transformations (connected to the identity). To see this explicitly, we perform a gauge transformation $A^{g}=g^{-1} A g+g^{-1} d g$ :

$C S\left(A^{g}\right)=C S(A)+\frac{k}{4 \pi} \int_{\partial \mathcal{M}} \operatorname{tr}\left(A \wedge d g g^{-1}\right)-\frac{k}{12 \pi} \int_{\mathcal{M}} \operatorname{tr}\left(d g g^{-1} \wedge d g g^{-1} \wedge d g g^{-1}\right)$.

The second term on the r.h.s. vanishes if $\mathcal{M}$ has no boundary or if $g$ vanishes at $\partial \mathcal{M}$. The third term also yields a boundary contribution only, as long as $g(x)$ is connected to the identity map in the gauge group. For arbitrary 'gauge transformations' $g(x)$ the CS-action picks up multiples of $2 \pi k$ in addition to boundary terms; however, for the standard choice $k$ an integer (times $\hbar$ ) such contributions do not contribute to the path integral.

It is important to note that the diffeomorphism symmetry of (6) is not independent from its non-abelian gauge symmetry. In fact one may easily verify the following identity [13]

$$
\mathcal{L}_{v} A=d\left(v^{\mu} A_{\mu}\right)+\left[A, v^{\mu} A_{\mu}\right]+\text { field equations },
$$

where $\mathcal{L}_{v}$ denotes the Lie derivative of the vector field $v$. This equations shows that on-shell an infinitesimal diffeomorphism may be generated by a gauge transformation with $g=1+v^{\mu} A_{\mu}+\ldots$, where the dots indicate terms of higher order. Correspondingly, at the Hamiltonian level of the theory there will be no independent additional first class constraints generating diffeomorphisms. Thus, in a theory like (6) it is sufficient to consider non-abelian gauge symmetries; diffeomorphisms (as well as frame-bundle rotations of the vielbein) are taken care of automatically.

We now wish to consider the case $\partial \mathcal{M} \neq 0$. It is therefore necessary to fix some boundary conditions on $\partial \mathcal{M}$. Introducing an auxiliary complex structure on this two-dimensional surface, let us, for concreteness, fix the $z$-component of $A:\left.\left(A_{z}\right)\right|_{\partial \mathcal{M}}:=\alpha$. With the addition of a boundary term $B[A] \equiv(k / 4 \pi) \int_{\partial \mathcal{M}} A_{\bar{z}} A_{z} d \bar{z} \wedge d z$ the variation of $C S[A]$ will have no unwanted 
boundary contributions. However, the total action $C S[A]+B[A]$ is not invariant under gauge transformations that do not vanish at $\partial \mathcal{M}$. This can quite easily be corrected by introducing group-valued fields $g$ living at the boundary. The total gauge-invariant action then takes the form

$$
\widetilde{C S}[A, g]=C S[A]+W Z N W[g]+\frac{k}{4 \pi} \int_{\partial \mathcal{M}} \operatorname{tr}\left(2 A_{z} \partial_{\bar{z}} g g^{-1}+A_{\bar{z}} A_{z}\right) d^{2} z
$$

where $W Z N W[g]$ is the action of the standard Wess-Zumino-Novikov-Witten model [30]:

$$
W Z N W[g]=\frac{k}{4 \pi} \int_{\partial \mathcal{M}} \operatorname{tr}\left(\partial_{z} g g^{-1} \partial_{\bar{z}} g g^{-1}\right) d^{2} z-\frac{k}{12 \pi} \int_{\mathcal{M}} \operatorname{tr}\left(d g g^{-1}\right)^{3} .
$$

According to Carlip and Balachandran et al, the $g$-dependent part of the action, i.e. the boundary action, must be quantized. The degeneracy of the resulting quantum theory should account for the statistical mechanical entropy of the black hole.

There are in fact several ways of deriving the above boundary action. The one chosen here, which followed primarily [6], is probably the shortest one. It is not, however, particularly instructive from a conceptual viewpoint. The reason is that we added the $g$-dependent terms in order to make the action gauge invariant. However, the WZNW-part of the action would not be needed to restore gauge invariance under those transformations that respect the boundary condition $\left.\left(A_{z}\right)\right|_{\partial \mathcal{M}}=\alpha$. One can therefore avoid the need for introducing edge dynamics by simply restricting to gauge transformations that respect the boundary conditions.

We will therefore sketch one further derivation of the boundary action in (9). Again we start with the CS-action (6). Now, however, we fix boundary conditions for $A_{z}$ only up to gauge transformations. To be more specific, we parametrize $A$ in terms of a quantity $\bar{A}$ that is fixed at the boundary and an unrestricted group-valued variable $g$ :

$$
A=g^{-1} \bar{A} g+g^{-1} d g .
$$

\footnotetext{
${ }^{5}$ Here we use an old trick: If an action behaves like $I\left(A^{g}\right)=I(A)+J(A, g)$ under gauge transformations $A \rightarrow A^{g}$, then clearly $I(A)+J(A, g)$ will be invariant under the simultaneous gauge transformations $A \rightarrow A^{h}$ and $g \rightarrow h^{-1} g$.

${ }^{6}$ The somewhat unconventional minus sign between the two terms may be changed by passing from $g$ to $g^{-1}$.
} 
Inserting this new parametrization into (6), we end up with the r.h.s. of Eq. (7) with bars on top of all the $A$ 's. For a well-defined variational problem we need to add a boundary term to kill the $\delta \bar{A}_{\bar{z}}$ contributions at $\partial \mathcal{M}$. For this purpose we could add, e.g., $(k / 4 \pi) \int_{\partial \mathcal{M}} \operatorname{tr}\left(\bar{A}_{\bar{z}}\left(\bar{A}_{z}+\partial_{z} g g^{-1}\right)\right) d^{2} z$. If, however, we require that everything added be expressible in terms of the original variables $A$ alone, i.e. in terms of the combination (11) of $\bar{A}$ and $g$, then the added term becomes unique and we end up with (9) with all the $A$ 's replaced by the gauge-fixed $\bar{A}$ 's. Note that by construction of (9) this is equivalent to $C S[A]+(k / 4 \pi) \int_{\partial \mathcal{M}} \operatorname{tr}\left(A_{z} A_{\bar{z}}\right) d^{2} z$ (without bars!) after using (11).

It is important to keep in mind that this second derivation leads to a significant interpretational shift, since we are now fixing $\bar{A}$ at the boundary instead of $A$ (which is equivalent to saying that we fix $A$ up to a gauge transformation). Choosing boundary conditions such that the 'threebeincomponents' of $\bar{A}$ correspond to a (possibly stretched) horizon, the threebeincomponents of $A$ will for general $g$ not correspond to a horizon. I Since $A$ is to be identified directly with the metrical variables, by adopting this second derivation we have to give up the picture that $\partial \mathcal{M}$ corresponds on-shell to a (stretched) horizon (of the classical spacetime solution). From this perspective, the variables $g$ now parametrize the 'deviation' of $\partial \mathcal{M}$ from the horizon, and this can become arbitrarily large now. Perhaps this is not unnatural: the relevant modes no longer "live on the boundary", but instead correspond to vibrational modes of the boundary itself.

This last derivation is in spirit very close to the one of Carlip. 8 However it is important to note that in order for this derivation to work, one must give up the notion that $\partial M$ corresponds to the horizon on-shell. This point is not made clear in Carlip's original paper 44. In fact, if one tries to implement Carlip's program without letting the location of the boundary "fluctuate off the horizon", one runs into the following problem: First fixing boundary con-

\footnotetext{
${ }^{7}$ It may be difficult to locate this in explicit coordinate calculations in the present context. This comes about since the BTHZ 'black hole' is just a quotient space of $2+1$ (anti-)deSitter space by some (properly discontinuously acting) discrete subgroup. As such any local patch looks completely alike. A 'horizon' can be defined only taking into account some nontrivial global issues. This changes drastically in the $1+1$ models considered below (or in the following Section). There the (Killing) horizon may be read off from the local value of the dilaton field and the above statement becomes completely evident in that case.

${ }^{8}$ And we gratefully acknowledge discussions on it with him.
} 
ditions for $A$, (e.g. fixing $A_{z}$ at $\left.\partial \mathcal{M}\right)$, then decomposing $A$ according to (11) and finally treating $\bar{A}$ as background currents that are 'fixed by the boundary data', would force the variable $g$ to be restricted at $\partial \mathcal{M}$ too. For example, if $\bar{A}_{z}$ is constant on $\partial \mathcal{M}$, then according to (11) $g$ may depend on $\bar{z}$ but not on $z$ and the WZNW-action would vanish trivially. In other words: Boundary data do indeed restrict the gauge symmetry at the boundary. However, by their nature they certainly also eliminate some of the original fields at the boundary (namely those fixed by the boundary data). In the present case these two mechanisms balance (the boundary data are a good cross section to the gauge symmetries at the boundary).

Irrespective of how one motivates or interprets the boundary contributions in (9), there still is another, mathematical question: The constraints following from (6) are of the form (2). It is not clear at this stage that the observables (3) are indeed in one-to-one correspondence with the group elements $g$ introduced as the edge degrees of freedom in (9), as assumed always in the literature.

Taking the $g$-dependent part of (9) as his starting point, Carlip [4] derives an entropy for the $2+1$ black hole [12] by counting quantum states in the edge model. In his earlier works this has been done for the Lorentzian signature of the metric. What he actually counted was rather the number of irreducible representations of the appropriate current algebra. In his latest work [5] Carlip redid the calculation for Euclidean signature, for which the theory can be formulated in terms of a compact gauge group and the quantization of the coupled WZNW model is therefore better defined. A key feature of Carlip's calculation was the need to impose a constraint on physical edge states. This constraint was roughly a remnant of the Wheeler-DeWitt equation for the bulk theory, and in the case of Lorentzian calculation at least, was needed to reduce the number of edge states to a finite number. In both the Lorentzian and Euclidean regimes, Carlip found the resulting entropy to coincide to leading order in $\hbar$ with the semiclassical Bekenstein-Hawking formula obtained by thermodynamical approaches [31].

Let us now turn to the simplest two-dimensional model, deSitter gravity [32. This model, characterized by $D=\Phi, V \propto \Phi$, and $Z \equiv 0$ in (4), may be formulated in terms of a non-abelian gauge theory of the BF-type [18]:

$$
L_{\mathrm{deS}}[A, B]=-2 \int_{\mathcal{M}} \operatorname{tr}(B F) .
$$

Here $F=d A+A \wedge A$ is the curvature two-form of a standard gauge field 
$A$, while $B$ is a Lie-algebra valued function on two-dimensional spacetime $\mathcal{M}$. The appropriate Lie algebras are $s o(2,1)$ and $s u(2)$ for Lorentzian and Euclidean signature of the metric theory, respectively (in the latter case this only holds only for an appropriate sign of the proportionality constant in $V$ ). Thus, e.g., $B=B^{i} T_{i}$ where $T_{i}$ are generators of the respective Lie algebra, normalized such that $-2 \operatorname{tr}\left(T_{i} T_{j}\right)=\delta_{i j}$ in the case of $s u(2)$, while for $s o(2,1)$ the normalization of $T_{1}$ is altered to $+2 \operatorname{tr}\left(T_{1} T_{1}\right)=1$. The identification of the metric variables with the gauge theory variables is similar to the one encountered in the 2+1-dimensional theory: The first two components of $A$ are identified with the zweibein while the last component corresponds to the spin connection. The first two components of $B$, on the other hand, are Lagrange multipliers enforcing torsion zero and $B^{3}$ turns out to be the dilaton $\Phi$. The action of diffeomorphisms on $A$ is again as in Eq. (8). An analogous equation may also be proven for $B$ so that the non-abelian gauge transformations again generate diffeomorphisms on-shell.

Next we determine the boundary action, following the simplest route. First let us agree to fix boundary conditions for $B$ and not for $A$ (for some motivation of this see below). Fixing $B$ (entirely or up to gauge transformations, as one prefers, cf. the discussion above), we should add a boundary term of the form $-\int_{\partial \mathcal{M}} \operatorname{tr}(B A)$. To make this gauge invariant with respect to gauge transformations restricted in no way at the boundary, we again should add group-valued edge variables. The appropriate action then is of the form

$$
\widetilde{L_{\mathrm{deS}}}[A, B, g]=-2 \int_{\mathcal{M}} \operatorname{tr}(B F)+2 \int_{\partial \mathcal{M}} \operatorname{tr}\left(B\left(A+d g g^{-1}\right)\right),
$$

which clearly is invariant under

$$
A \rightarrow A^{h}=h^{-1} A h+h^{-1} d h, \quad B \rightarrow B^{h}=h^{-1} B h, \quad g \rightarrow g^{h}=h^{-1} g
$$

for arbitrary maps $h(x)$ from $\mathcal{M}$ to $G$.

Before turning to the quantization of the boundary action, some remarks are in order: First, on-shell the two components $B^{a}$ of $B$ coincide with a Killing vector field $k^{a}$ in an orthonormal frame bundle basis. Actually a similar statement holds for all the theories (14), which locally always have (at least) one Killing field $k$ (cf., e.g., [21, 27]). The horizon of a black hole thus coincides with lines where $(k)^{2} \equiv B^{a} B_{a}=0$. For Lorentzian signature of (四), the horizon may therefore be characterized by $B^{+} \equiv\left(B^{1}+B^{2}\right) / \sqrt{2}=0$ and/or $B^{-} \equiv\left(-B^{1}+B^{2}\right) / \sqrt{2}=0$. The bifurcation point where the past and 
future horizon of an eternal black hole coincide is specified by $B^{+}=B^{-}=0$, which is a hyperbolic fixedpoint of $k$ in this case. For Euclidean signature, on the other hand, the horizon degenerates to a point, $B^{1}=B^{2}=0$, which now is an elliptic fixed point of $k$.

The black hole mass, furthermore, may be identified, on-shell, with the gauge-invariant combination

$$
M=-2 \operatorname{tr}(B \cdot B) \equiv B^{a} B_{a}+\left(B^{3}\right)^{2},
$$

where $B^{a} B_{a}$ equals $2 B^{+} B^{-}=-\left(B^{1}\right)^{2}+\left(B^{2}\right)^{2}$ in the Lorentzian and $\left(B^{1}\right)^{2}+$

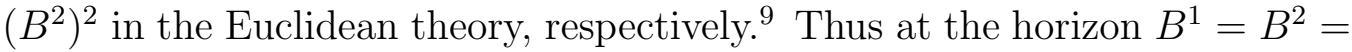
0 and $B^{3} \equiv \Phi=\sqrt{M}$, while at a 'stretched horizon' the $B^{i}$ would be close to these values.

This simple option to enforce ones boundary to coincide with a (stretched) black hole horizon may be seen as a motivation for choosing to fix $B$ at the boundary $\partial \mathcal{M}$. In this context it is then also particularly clear that if we adopt the viewpoint that $B$ at $\partial \mathcal{M}$ is fixed only up to gauge transformations (i.e. $B_{\partial \mathcal{M}}=\left(g^{-1} \bar{B} g\right)^{3}$ with $\bar{B}^{a}=0$ and $\left.\left.\left(\bar{B}^{3}\right)\right|_{\partial \mathcal{M}}=\sqrt{M}\right)$ then $\left.\left(B^{3}\right)\right|_{\partial \mathcal{M}}$ may take any value depending on the choice of $g$ and the boundary will be a horizon only for $g$ in the stabilizer $H$ of the Lie algebra element $T_{3}$. In this picture $g \in G / H$ is found to precisely determine the location of (a point on) $\partial \mathcal{M}$ (at a given time) within the boundaryless maximal extension of $\mathcal{M}$. We believe that this feature is not an artifact of the two-dimensional model at hand, but that it is generic and present also, e.g., in the $2+1$ theory, only there it is less obvious due to the lack of $B$-fields (cf. also the remarks in footnote 8).

Note also that if we fixed boundary conditions merely for the $A$ 's, we would induce no boundary terms in (12), which moreover is already gauge invariant. On the other hand, the constraints would be of the form (2) and observables of the form (3) should exist. A similar discrepancy may be observed also in the case of an abelian $B F$-action even when treating boundary conditions for the $B$-field (cf. also the remarks in the Outlook).

Following [4, 6], we now need to quantize

$$
L_{\text {coad }}=2 \int_{\partial \mathcal{M}} \operatorname{tr}\left(B d g g^{-1}\right)
$$

\footnotetext{
${ }^{9}$ The appropriate generalization of this for the general nonlinear gauge theory (4) will be dealt with in the next section.
} 
where $B$ should be thought of as a fixed element in the Lie algebra. This is quite straightforward and was solved some time ago [33, 34]. To keep this work self-contained and to keep the mathematical aspects of the 2-D model as transparant and simple as possible, we now give a brief account of the quantization of (16), referring to the Appendix for further details.

We start with a simultaneous treatment of the Lorentzian and Euclidean signature, corresponding to $G=\widetilde{S O}_{e}(2,1)$ (cf. last reference in 18]) and $G=S U(2)$, respectively, where $\widetilde{S O_{e}}(2,1)$ denotes the universal covering group of the component of $S O(2,1)$ connected to the identity. let us align $B$ with the three-direction in the Lie algebra: $B=\sqrt{M} T_{3}, M$ constant. Any other choice of boundary conditions for $B$, at least if they are constant along $\partial \mathcal{M}$ and if $M>0$ in (15) certainly, may be mapped to this problem by an appropriate change of variables in (16). Thus the point particle action $L_{\text {coad }}$ reduces to

$$
L_{\text {coad }}=2 \sqrt{M} \int \operatorname{tr}\left(T_{3} \dot{g} g^{-1}\right) d t
$$

where $t$ denotes the coordinate along $\partial \mathcal{M}$. This action is already in first order form and basically coincides with an action $\int p \dot{q} d t$. The Hamiltonian of the system is zero (strongly, not only weakly), as it should be for a diffeomorphism invariant system. (Note that we have not broken the diffeomorphism invariance along the boundary; this could occur only, if the boundary conditions for $B$ were chosen to be explicitly time-dependent). It still remains to determine the phase space topology (space of values for $q$ and $p$ ), which will turn out to be nontrivial.

Since $g$ takes values in a three-dimensional group $G$, the phase space can be at most of dimension two. Indeed, parametrizing $g$ according to $g=l \tilde{g}$, where $l=\exp \left(\lambda T_{3}\right) \in H, H$ being the one-dimensional stabilizer subgroup of $B \propto T_{3}$, so that $\tilde{g} \in G / H$, we find

$$
L_{\text {coad }}=2 \sqrt{M} \int\left[\operatorname{tr}\left(T_{3} \dot{\tilde{g}} \tilde{g}^{-1}\right)-\frac{1}{2} \dot{\lambda}\right] d t .
$$

So, the one dimension along $H$ drops out from the action as a total divergence. The phase space of the point particle at the boundary is found to be $G / H$, endowed with the (Kirrillov) symplectic two-form

$$
\Omega=2 \sqrt{M} \operatorname{tr}\left(T_{3} d \tilde{g} \tilde{g}^{-1} \wedge d \tilde{g} \tilde{g}^{-1}\right)
$$

\footnotetext{
${ }^{10} \widetilde{S O_{e}}(2,1)$ has no finte-dimensional faithful matrix representation; so the trace in $(16)$ is to be replaced by the Killing metric in this case.
} 
In order to complete the calculation, we must now specify the signature. We consider the Euclidean theory first. In this case $G=S U(2), H=$ $U(1)$ and $G / H=S^{2}$ (the famous Hopf fibration of $S U(2) \sim S^{3}$ ). We can parametrize $G$ as

$$
g=\left(\begin{array}{cc}
\cos \frac{\theta}{2} e^{i(\varphi-\lambda) / 2} & \sin \frac{\theta}{2} e^{-i(\varphi+\lambda) / 2} \\
-\sin \frac{\theta}{2} e^{i(\varphi+\lambda) / 2} & \cos \frac{\theta}{2} e^{-i(\varphi-\lambda) / 2}
\end{array}\right)
$$

with $\theta \in[0, \pi], \varphi \in[0,2 \pi]$, and $\lambda \in[0,4 \pi]$. Here $\theta$ and $\varphi$ become the standard spherical variables of the phase space $S^{2}$, while $\lambda / 2$ is the angular variable along the diagonal subgroup that was found to drop out.⿴囗十 Furthermore, it is straighforward to verify that $p:=\sqrt{M} \cos \theta$ and $q:=\varphi$ provide Darboux coordinates, i.e. in these coordinates $\Omega=d p \wedge d q$. Certainly this equation holds locally only, everywhere at the two-sphere except for its poles $p= \pm \sqrt{M} ; \Omega$, being proportional to the volume-form, is closed but not exact.

A system with a compact two-dimensional phase space may be quantized only if $\oint \Omega=2 \pi n \hbar, n \in Z$ ((generalized) Bohr-Sommerfeld quantization condition, cf. also the Appendix and, e.g., [34]). This yields the consistency condition:

$$
M=n^{2} \hbar^{2} / 4
$$

Thus, it is only for these values of the mass $M$ (up to a possible shift, cf. the discussion in the Appendix) that a consistent quantization of the boundary action can be carried out. The constraint (20) may also be obtained by requiring (16) to become single-valued on $G / H \sim S^{2}$ up to multiples of $2 \pi \hbar$. In fact the action $L_{\text {coad }}$ is of the Wess-Zumino type, analogous to the second term in (10), an observation that will become more transparent in the formulation of the following section. Here in view of (18) this may be verified by noting that $\lambda / 2 \sim \lambda / 2+2 \pi$, which again enforces (20) for consistency.

In the above the parameter $M$ entered as a classical quantity, coinciding with the mass of the classical spacetime solution. If, on the other hand, we quantize the gravitational field by quantizing the $B F$ bulk action, $M$ becomes an operator on the space of physical wavefunctionals $\Psi[A]$ or $\Psi[B]$. It is therefore reassuring that the spectrum of the corresponding quantum

\footnotetext{
${ }^{11}$ We are using the representation $T_{i}=-i \sigma_{i} / 2$, where $\sigma_{i}$ are the standard Pauli matrices.
} 
operator $M$ coincides with the one found in (20) (up to possibly precisely the shift(s) discussed in the Appendix, cf. the ongoing discussion on the issue of the spectrum of $\operatorname{tr}(B \cdot B)$ in, e.g., [35]): In the connection representation $\Psi[A]$, gauge invariance of the physical wave functionals restricts $\Psi$ to live on the Weyl cell of $s u(2)$, which is just an interval along $T_{3}$. The Casimir $M \propto \operatorname{tr}(B)^{2}$, on the other hand, becomes nothing but the Laplacian on the Weyl cell; clearly here (and similarly for other compact gauge groups) this yields a discrete spectrum for $M$.

The next step in Carlip's program is to determine the number $N$ of quantum states for the edge degrees of freedom living on the boundary of a spacetime with mass $M$ satisfying (20). Clearly this will be finite because the phase space is compact. This is quite satisfactory since no regularization will be required when calculating the statistical mechanical entropy via $S_{\text {st.m. }}=k \ln N$.

An approximate answer for the degeneracy of states can be obtained immediately. From elementary statistical mechanics we know that there will be about one quantum state per phase space volume $h$. Since the total symplectic volume was found to be $n h$, this leads to $N \sim n$. In the Appendix we present three precise calculations by applying three different quantization procedures to the present system. Two of these lead to $N=n$, one to $N=n+1$. This difference is not crucial for our purposes, and we will stick to $N=n$ in what follows. Thus we may conclude

$$
S_{\text {st.m. }} \propto \ln n=\ln (2 \sqrt{M} / \hbar) .
$$

Unfortunately this result does not match the thermodynamical entropy for this model using other methods [21, 15]. For example, Wald's general method starts with the variation of the action under spacetime diffeomorphisms of the form:

$$
\begin{aligned}
x^{\mu} \rightarrow x^{\prime \mu} & =x^{\mu}+\delta x^{\mu} \\
\Phi^{A}(x) \rightarrow \Phi^{\prime A}\left(x^{\prime}\right) & =\Phi^{A}(x)+\delta \Phi^{A}(x),
\end{aligned}
$$

where for the moment we use a condensed notation in which the complete set of fields (including the metric) is denoted by $\Phi^{A}(x)$, and $x$ is the spacetime coordinate. Under such a general transformation, an action which is second order in derivatives of the fields has the following variation:

$$
\delta I=\int d^{2} x\left(\frac{\delta I}{\delta \Phi^{A}} \delta \Phi^{A}+\frac{\partial j^{\mu}}{\partial x^{\mu}}\right)
$$


where $j^{\mu}$ is the associated Noether current. Diffeomorphism invariance of the action requires that the Noether current be divergence free when the classical field equations are satisfied.

For the action (4) in a parametrization for which $D(\Phi)=\Phi$ and $Z(\Phi)=0$, the Noether current is [36]:

$$
\begin{aligned}
j^{\lambda}= & (\Phi R-V) \delta x^{\lambda}-\nabla_{\sigma} \Phi\left(g^{\alpha \lambda} g^{\beta \sigma}-g^{\alpha \beta} g^{\lambda \sigma}\right) \bar{\delta} g_{\alpha \beta} \\
& +\Phi\left(g^{\alpha \sigma} g^{\beta \lambda}-g^{\alpha \beta} g^{\sigma \lambda}\right) \nabla_{\sigma}\left(\bar{\delta} g_{\alpha \beta}\right),
\end{aligned}
$$

where $\bar{\delta}$ denotes variation of the corresponding field under Lie derivation along $\delta x^{\mu}$. As remarked already above, all the solutions have a Killing vector $k$. One can show that it may be written as

$$
k^{\lambda}=-\epsilon^{\lambda \sigma} \nabla_{\sigma} \Phi .
$$

Choosing $\delta x^{\lambda}:=-k^{\lambda}$, the variations of the scalar field and metric will vanish on-shell for such transformations, and the Noether current becomes:

$$
j^{\lambda}=\left(\Phi \frac{d V}{d \Phi}-V\right) \epsilon^{\lambda \sigma} \nabla_{\sigma} \Phi,
$$

where we made use of the field equation $R=V^{\prime}(\Phi)$. Clearly the equation $\partial_{\mu} j^{\mu}=0$ implies that the Hodge dual of $j, J_{\mu}=\epsilon_{\mu \lambda} j^{\lambda}$ is closed (on-shell) and thus locally exact:

$$
J=\left(V-\Phi V^{\prime}(\Phi)\right) d \Phi=d Q
$$

By means of the field equations one may verify, furthermore, that the Noether charge $Q$ associated with the Killing vector is:

$$
Q=V \Phi-g^{\alpha \beta} \nabla_{\alpha} \Phi \nabla_{\beta} \Phi
$$

According to Wald's prescription, the black hole entropy should be $2 \pi$ times the Noether charge associated with the Killing vector whose norm vanishes on the horizon, providing that the Killing vector is normalized to have unit surface gravity. The normalization condition effectively requires us to divide by the surface gravity $\kappa$ of the black hole, so that

$$
S=\frac{\left.2 \pi Q\right|_{\text {horizon }}}{\hbar \kappa}
$$


A straightforward calculation [21] gives the surface gravity of a black hole in generic dilaton gravity to be:

$$
\kappa^{2} \equiv-\frac{1}{2} \nabla^{\mu} k^{\nu} \nabla_{\mu} k_{\nu}=\left(\frac{\left.V\right|_{\text {horizon }}}{2}\right)^{2} .
$$

Moreover, on the horizon, according to (25), $|\nabla \Phi|^{2}=0$, so that the final expression for the entropy is:

$$
S=\frac{\left.4 \pi \Phi\right|_{\text {horizon }}}{\hbar}
$$

More generally, this procedure yields Eq. (5). For the specific case at hand (i.e. $V(\Phi) \propto \Phi=D(\Phi)$ and $Z=0) \Phi=B^{3}=\sqrt{M}$ on-shell and

$$
S_{\text {thermo }} \propto \sqrt{M} .
$$

We now briefly comment on the Lorentzian signature case, in which the coadjoint orbit will be non-compact and the total symplectic volume is divergent. Correspondingly, there will be infinitely many quantum states leading to a meaningless $N=\infty$. Also there will be no consistency condition enforcing a discrete spectrum for $M$; for any value of $M$ there exists a Hilbert space (of the edge states) which is infinite dimensional. We will discuss this case further in what follows.

\section{The General Model}

We now apply the method of the previous section to the generalized dilaton theories of Eq.(4). This constitutes a qualitatively new step, as these theories do not in general allow for a (standard) gauge theory formulation. However, the notion of Poisson $\sigma$-models is applicable and will make these models tractable in the present context. For simplicity we will for the most part restrict ourselves to the case $Z \equiv 0, D=\Phi$ in what follows.

The model (4) may be described by [27]:

$$
L\left[A_{i}, X^{i}\right]=\int_{\mathcal{M}} A_{i} \wedge d X^{i}+\frac{1}{2} \mathcal{P}^{i j}(X(x)) A_{i} \wedge A_{j}
$$

with

$$
\left(\mathcal{P}^{i j}\right)(X) \equiv\left(\begin{array}{ccc}
0 & -V\left(X^{3}\right) / 2 & -X^{2} \\
V\left(X^{3}\right) / 2 & 0 & \pm X^{1} \\
X^{2} & \mp X^{1} & 0
\end{array}\right) \quad i, j \in\{1,2,3\}
$$


where the upper sign corresponds to the Euclidean, the lower sign to the Lorentzian signature of the theory. In this context one can regard $A$ as a triple of one-forms and $X$ as a triple of functions on spacetime. This is in fact a generalization of the situation encountered in the BF-theory (12) discussed above (with the obvious change of notation from $B$ to $X$ ). The identification with geometrical variables is therefore as follows: The first two components of $A$ coincide with the zweibein, the last one with the spin connection. $X^{1}$ and $X^{2}$ are Lagrange multipliers enforcing zero torsion whereas $X^{3} \equiv \Phi$. (For more general $D$ and/or $Z$ cf. [27, 37]).

The Lie algebra of the gauge group is now replaced by the Poisson bracket $\left\{X^{i}, X^{j}\right\} \equiv \mathcal{P}^{i j}(X)$ on the target space $\mathbb{R}^{3}$ spanned by the three linear target space coordinates $X^{i}$. The latter reduces to an ordinary Lie algebra, if $\mathcal{P}^{i j}$ is linear in $X$. For $\mathcal{P}^{i j}=\varepsilon^{i j}{ }_{k} X^{k}$ we recover the previous $B F$-theory after a partial integration. In fact, the boundary term picked up by the partial integration is nothing but the one that we added in the course of our derivation of (13); thus (33) is already of the appropriate form for boundary conditions on $X$ - up to the gauge transformations, possibly, which will now be discussed.

Making use of the central relation $\left(\partial \mathcal{P}^{i j} / \partial X^{s}\right) \mathcal{P}^{s k}+$ cycl. $(i j k)=0$ (the Jacobi identity for the bracket $\left.\left\{X^{i}, X^{j}\right\}\right)$, it is easy to verify that under

$$
\delta_{\epsilon} X^{j}=\epsilon_{i}(x) \mathcal{P}^{i j}, \quad \delta_{\epsilon} A_{i}=d \epsilon_{i}+\mathcal{P}^{l m}{ }_{i} A_{l} \epsilon_{m}
$$

the action (33) changes only by a total divergence:

$$
\delta_{\epsilon} L=\int_{\partial \mathcal{M}} X^{i} d \epsilon_{i}
$$

The symmetries (35) are an obvious generalization of the standard nonabelian gauge symmetries (which arise for linear $\mathcal{P}$ ). By an appropriate choice of $\epsilon_{i}$ (35) may also generate diffeomorphisms: For $\epsilon_{i}:=A_{i \mu} v^{\mu}$ one verifies $\delta_{\epsilon}=$ $\mathcal{L}_{v}+$ (terms vanishing by means of the field equations), which generalizes (8) to the present context.

Following the first route for obtaining (9), we fix $X$ at $\partial \mathcal{M}^{\mathbb{4} 3}$ and add (36) to (33), with $\epsilon_{i}$ now denoting additional fields, the 'edge degrees of freedom'.

\footnotetext{
${ }^{12} X^{i}(x)$ denotes the map from the spacetime $\mathcal{M}$ to this 'target space', the space of values for the fields $X^{i}$. Although this space is $\mathbb{R}^{3}$ and thus linear here, it carries a non-linear structure given by the two-tensor $\mathcal{P}^{i j}$. For more details see [25, 26, 27], in particular the last two of them for a pedagogical introduction.

${ }^{13}$ This could be motivated by remarking that everything written in the two paragraphs surrounding Eq. 15 is valid here too with the replacement $B \rightarrow X$.
} 
However, for $\left.\left(X^{i}\right)\right|_{\partial \mathcal{M}}:=$ const the (36) becomes a total divergence and there is no action for the boundary degrees of freedom. What did we do wrong? After all we know, e.g., that for the special case (12) of (33) there does exist a nontrivial action for the boundary variables.

The solution to this apparent puzzle stems from the fact that the symmetries (35) are written in their infinitesimal form, which is not sufficient here. Upon inserting $g:=1+\epsilon(x)$ into (16) and keeping only first order terms in $\epsilon$, one also is left with a (meaningless) total divergence on $\partial M$ only. This illustrates the main difficulty in applying the approach of the preceeding section to more general models; usually one knows the symmetries generated by some constraints only in their infinitesimal form, while the diffeomorphism group is difficult to handle because it is infinite dimensional and acts 'nonlocally' on the fields. In the present case it is also far from trivial to 'exponentiate' the local symmetries (35).

At this point we apply a trick that is standard in the framework of Poisson $\sigma$-models: We change the parametrization of the target space of the theory and use coordinates on it which are particularly adapted to the Poisson tensor $\mathcal{P}$. In particular, we change field variables $X^{i} \rightarrow \widetilde{X}^{i}$ with

$$
\widetilde{X}^{i}:=\left(X^{a} X_{a}-\int^{X^{3}} V(z) d z,\left\{\begin{array}{c}
\arctan \left(X^{2} / X^{1}\right) \\
\operatorname{artanh}\left(X^{2} / X^{1}\right)
\end{array}\right\}, X^{3}\right)
$$

where $X^{a} X_{a}= \pm\left(X^{1}\right)^{2}+\left(X^{2}\right)^{2}$ and the upper/lower line of the second entry in (37) corresponds to the upper/lower sign in (34), i.e. to the signature of the theory. In terms of the new variables, the two-tensor $\mathcal{P}$ takes the form

$$
\left(\widetilde{\mathcal{P}^{\widetilde{i j}}}\right)(\widetilde{X})=\left(\begin{array}{ccc}
0 & 0 & 0 \\
0 & 0 & 1 \\
0 & -1 & 0
\end{array}\right)
$$

which simplifies $(33)$ to

$$
L=\int_{\mathcal{M}} \widetilde{A}_{i} \wedge d \widetilde{X}^{i}+\widetilde{A}_{2} \wedge \widetilde{A}_{3}
$$

(Here we used the obvious target space covariance of (33). In particular, $\left.\widetilde{A}_{i} \equiv\left(\partial X^{j} / \partial \widetilde{X}^{i}\right) A_{j}\right)$.

The parametrization $\widetilde{X}, \widetilde{A}$ of the field variables of $L$ generically holds only on some local patch. This is, however, sufficient for our purposes; there 
are further 'charts' on $\mathbb{R}^{3}$ beside (37) bringing $\mathcal{P}$ into the 'Casimir-Darbouxform' (38) and such charts may be patched together. In any of these local charts the symmetries (35) now take the form:

$$
\delta \widetilde{A}_{i}=d \widetilde{Y}^{i}, \quad \delta \widetilde{X}^{1}=0, \delta \widetilde{X}^{2}=\widetilde{Y}^{3}, \delta \widetilde{X}^{3}=-\widetilde{Y}^{2} .
$$

Here the three fields $\tilde{Y}^{i}$ may already be considered as finite quantities, since in this form the symmetries are linear in the parameter fields. Note that this form of the action and symmetries even simplifies the group theoretical case (12).

Now we look at the (non-infinitesimal) change of $L$ in this parametrization of the symmetries. Again it is a surface term, but now one that is non-trivial:

$$
\delta L=\int_{\partial \mathcal{M}}\left(\widetilde{X}^{i} d \widetilde{Y}^{i}+\widetilde{Y}^{2} d \widetilde{Y}^{3}\right) .
$$

Given our boundary conditions on the fields $X$, we may again drop the first term in the above. What we are left with is the edge state action

$$
L_{\text {Symp }}\left[\widetilde{Y}_{2}, \widetilde{Y}_{3}\right]=\int_{\partial \mathcal{M}} \widetilde{Y}_{2} \wedge d \widetilde{Y}^{3},
$$

which is already of the simple form $\int p \dot{q} d t$ (after identifying $\tilde{Y}^{3}$ with a 'generalized coordinate' $q$ and $\tilde{Y}^{2}$ with a 'generalized momentum' $p$ ). Thus we did not have to find new coordinates such as $\theta$ and $\varphi$ in (19) in order to obtain the point particle action at the boundary in Darboux-form; it appeared in this form directly as a result of introducing coordinates (37) adapted to the Poisson structure (34). It is also worth noting that it was the contribution of the $\widetilde{A} \wedge \widetilde{A}$ term that gave the nontrivial result (42) within the present formulation; the surface term $-\int_{\partial \mathcal{M}} \widetilde{X}^{i} \widetilde{A}_{i}$ did not contribute at all.

$L_{\text {symp }}$ is the analogue, or better generalization, of the local $p \dot{q}$-form of the action (16). Once we are equipped with some geometrical insight into the 'coordinates' $\widetilde{X}^{i}$, we will be able to interpret $L_{\mathrm{Symp}}$ globally. According to (38) it is $\widetilde{X}^{1}$, a Casimir coordinate of the Poisson bracket on the target $\mathbb{R}^{3}$, that determines the symplectic leaves, as previously mentioned in the Introduction. Furthermore, $\widetilde{X}^{2}$ and $\widetilde{X}^{3}$ serve as Darboux coordinates on any of these leaves $\widetilde{X}^{1}=$ const. Note that, as an obvious consequence of (39), $\widetilde{X}^{1}(x)$ must also be a constant on the spacetime. Thus the map from $\mathcal{M}$ into the target $\mathbb{R}^{3}$ has to lie completely within a symplectic leaf. It turns out, moreover, that the corresponding value of $\widetilde{X}^{1}$ on a classical solution may be 
identified with the mass $M$, or at least a function of $M$, whenever the notion of mass makes sense (cf., e.g., [38]).

In view of the last two equations (40) it is now clear that the fields $\tilde{Y}^{2}$ and $\tilde{Y}^{3}$ should take values not just in an $\mathbb{R}^{2}$, as they do locally, but in the same space as the Darboux coordinates mentioned above. That is, they should also be regarded as local coordinates of a symplectic leaf. This symplectic leaf is a copy of the one of described by $\widetilde{X}^{2}$ and $\widetilde{X}^{3}$, which is determined by the value of the Casimir $\widetilde{X}^{1} \sim M$. Similary one can introduce three linear coordinate fields $Y$ and describe the symplectic leaf under consideration by

$$
Y^{a} Y_{a}-\int^{Y^{3}} V(z) d z=M
$$

where $M$ is the mass specified by the boundary conditions on $X$.

We are now in a position to present the correct total gauge-invariant action for the general nonlinear system (33):

$$
\widetilde{L}[A, X, Y]=L[A, X]+L_{\text {Symp }}[M[X], Y],
$$

where the fields $Y \in \mathbb{R}^{3}$ are subject to (43). It is the appropriate generalization of (13). The coadjoint orbit encountered in (16) is generalized to a symplectic leaf. A (target space) coordinate independent description of $L_{\text {symp reads }}$

$$
L_{\text {symp }}=\left.\int_{\partial \mathcal{M}} d^{-1} \Omega\right|_{M}
$$

where $\Omega$ denotes the symplectic two-form on the symplectic leaf specified by the value of $M=\widetilde{X^{1}}$ (in the above coordinates $\Omega=d \widetilde{Y}^{2} \wedge d \widetilde{Y}^{3}$ ). More precisely, $\Omega$ is the symplectic two-form of the Poisson bracket (34) with $X \rightarrow$ $Y$ and $Y \in \mathbb{R}^{3}$ subject to (43).

The symbol $d^{-1} \Omega$ has been used to denote a symplectic potential (oneform), such as the one used in (42). However, if the second homotopy group $\pi_{2}$ of the target leaf is not trivial, such a potential will exist only locally. But the action should be independent of the particular local trivialization. The situation is very similar to the WZNW-action (10); the second term in (44) may also be written as $(k / 12 \pi) \int_{\partial \mathcal{M}} d^{-1}\left(d g g^{-1}\right)^{3}$, and single-valuedness of the quantum action (path integral) restricts the "coupling constant". Here the role of the latter is played by the mass $M$. For example, suppose $\partial \mathcal{M}=S^{1}$ in the present case. Then the right hand side of (45) can be replaced by 
$\left.\int_{\mathcal{M}} \Omega\right|_{M}$, which is independent (up to multiples of $2 \pi$ ) of how the integral is continued from its boundary values if and only if

$$
\left.\oint \Omega\right|_{M}=2 \pi n \hbar,
$$

$n \in Z$. For $\pi_{2}$ (leaves) $\neq 0$ this restricts the possible values of $M$, a mechanism that we encountered already in a particular case when dealing with the BFtheory.

Eq. (46) can also be obtained as a necessary and sufficient condition for the geometric quantization of the phase space (43) with symplectic form $\left.\Omega\right|_{M}$ (cf. the Appendix). Precisely the same condition arises in the Dirac quantization of the bulk action as the global integrability condition of the quantum constraints, whose local integrability is ensured from the closure of the quantum constraint algebra [25]. The question of continuity or discreteness for the spectrum of $M$ is reduced to a simple, purely topological question: The mass spectrum is discrete, iff the two-surfaces generated by (43) have nontrivial second homology. Alternatively: A discrete spectrum of $M$ (within the range of values $[a, b]$ ) occurs, iff all the two-surfaces (within the range $M \in[a, b])$ are compact. In view of (43) it is easy to see that a discrete spectrum can occur only for the Euclidean signature theory, but even in that case it is the exception.

At this point let us remark that for the case of a non-trivial first homotopy of the surfaces (43) of constant $M$, we believe that the above picture has to be corrected somewhat. The phase space of the edge particles should then rather be regarded as the universal covering of the level surfaces of $M$ (with the symplectic form given by the pull-back of $\Omega$ under the covering map). The origin for this slight modification comes from large gauge transformations and is basically the same as the one that, in the special case of Lorentzian deSitter gravity, makes $\widetilde{S O}_{e}(2,1)$ the correct gauge group rather than $S O_{e}(2,1)$.

We are left with the task of determining the number of quantum states on $\partial \mathcal{M}$. Here we will content ourselves with a number of general statements and then briefly focus on spherically symmetric and string inspired dilaton gravity for illustration. The number of quantum states of the mechanical model described by $L_{\mathrm{Symp}}$ is finite, iff the two-surface (43) (its phase space) is compact. As found above in this case the mass is discretized. For any one of these allowed values, $M=M_{n}$ such that (46) is satisfied, the number of

\footnotetext{
${ }^{14}$ We remark that in the approach of [39] one obtains a purely discrete mass spectrum for all theories (4) with Euclidean signature.
} 
quantum states may be approximated very well by $n$ (as follows from the same argument used in the preceeding section or by the more precise consideration in the footnote of the Appendix). Still only in exceptional cases the entropy obtained via $S=S_{\text {st.m. }} \propto \ln (n)$ will fit the thermodynamical $S=S_{\text {thermo }}$ given in (5).

Actually, we can be even more concrete on this point. A compact leaf is obtained, iff there are two successive zeros of the function $M+\int{ }^{Y^{3}} V(u) d u$ (as a function of $Y^{3}$ ) between which this function is positive. (Note that due to (43) this expression equals $\left.Y^{a} Y_{a}=\left(Y^{1}\right)^{2}+\left(Y^{2}\right)^{2}\right)$. Denote the corresponding two values of $Y^{3}$ by $\left(Y^{3}\right)_{>}$and $\left(Y^{3}\right)_{<}$, respectively, (with $\left.\left(Y^{3}\right)_{>}>\left(Y^{3}\right)_{<}\right)$. According to our knowledge of the symplectic form the integral over $\Omega$ is computed easily (just use Darboux coordinates, cf. Eq. (37)):

$$
\oint \Omega=2 \pi\left[\left(Y^{3}\right)_{>}-\left(Y^{3}\right)_{<}\right] .
$$

Together with (46) and $S_{\text {st.m. }} \propto \ln n$ this then yields

$$
S_{s t . m .} \propto \ln \left(\left[\left(Y^{3}\right)_{>}-\left(Y^{3}\right)_{<}\right] / \hbar\right)
$$

for the case of compact phase spaces. In general this disagrees with (5). Agreement would be found only for the (almost pathological) case $\left(Y^{3}\right)_{<}=$ $-\exp \left(\left(Y^{3}\right)_{>}\right)+\left(Y^{3}\right)_{>}$(at least if one takes serious (5) also for the case of several horizons). In the instance of the previous section we had $\left(Y^{3}\right)_{>}=$ $-\left(Y^{3}\right)_{<}=\sqrt{M}$ and (48) reduces to (21), as it should.

In all the cases where the surfaces (43) are non-compact there will be an infinite number of edge states. At least in cases where these surfaces have trivial topology $\mathbb{R}^{2}$, there seems to be no sensible way to extract a finite number from that quantization. For phase space topology $\mathbb{R}^{2}$ we know that there exist coordinates $q, p$ on phase space such that $\Omega=d p \wedge d q$ globally (Darboux theorem). If, furthermore, the respective symplectic leaf is complete with respect to the flow of Hamiltonian vector fields, this in turn implies that the quantization of the system is unique up to unitary equivalence. This is most easily seen by noting that then the transition to coordinates $q, p$ maps the system under consideration to the one of an ordinary point particle on the line. So here we cannot, for example, do as Carlip did in Lorentzian $2+1$ gravity, and count the number of irreducible representations of the operator algebra to be quantized. This kind of 'regularization' would yield a meaningless one in the present context. 
As an illustration of these statements let us finally specialize to ordinary dilaton gravity (the so-called "string-inspired" model) as well as to spherically reduced gravity [20]. Both of these models are governed by an action of the type (田), albeit not of the particular form with, e.g., $Z \equiv 0$ to start with. The appropriate expressions replacing (43) in these cases are

$$
M_{\text {dil }}=\frac{Y^{a} Y_{a}}{4 \lambda}+\lambda Y^{3}, \quad M_{\mathrm{SS}}=-Y^{a} Y_{a}+\sqrt{Y^{3}},
$$

where $\lambda>0$ and $Y^{3}$ is restricted to positive values and. Clearly (49) picks out non-compact surfaces for Lorentzian signature, $Y^{a} Y_{a}=-\left(Y^{1}\right)^{2}+\left(Y^{2}\right)^{2}$ (as is always the case with expression of the form (43)). Unfortunately, it does so here too for Euclidean signature, $Y^{a} Y_{a}=\left(Y^{1}\right)^{2}+\left(Y^{2}\right)^{2}$. Moreover, for any value of the mass $M$ these surfaces are simply connected and diffeomorphic to $\mathbb{R}^{2}$. In the Euclidean case they are also complete. ${ }^{\text {Pr }}$ Thus, for reasons discussed above, the Hilbert space of the edge system coincides uniquely with the one of square integrable functions on the line $\mathbb{R}$. In the Lorentzian case the leaves are not complete with respect to the flow of Hamiltonian vector fields fields). There are, however, still infinitely many quantum states associated with these phase spaces since the total symplectic volume diverges (in the Lorentzian case the quantization is similar to the one of a particle restricted to an interval).

Thus we get infinitely many edge states in the Lorentzian and the Euclidean theories here. As with most 2-D models, therefore, we cannot use the present approach to calculate a statistical mechanical entropy for these two prominent cases.

\section{Discussion and Outlook}

We have attempted to calculate the statistical mechanical entropy for black holes in $2 D$ generic dilaton gravity in terms of edge states that arise when the

\footnotetext{
${ }^{15}$ For ordinary dilaton gravity, e.g., this is most obvious by noting that $Y^{1}$ and $Y^{2} / 2 \lambda^{2}$ are possible Darboux coordinates on $M_{\text {dil }}=$ const $>0$ and that their range is all of $\mathbb{R}^{2}$ on these surfaces.

${ }^{16} \partial / \partial Y^{3}$, e.g., is the Hamiltonian vector field of $\ln Y^{+}$and thus is incomplete with respect to its flow parameter since $Y^{3}>0$ (on-shell $X^{3}$, of which $Y^{3}$ is a copy, equals the exponential of the dilaton field $\Phi$ or the square of the Schwarzschild coordinate $r$, for the string inspired and the Schwarzschild case, respectively).
} 
event horizon is treated as a boundary to spacetime. We discussed various routes suggested in the literature for obtaining the action governing these boundary modes.

Since none of these seemed compelling to us, we suggested a modified reasoning where the boundary does not coincide with the event horizon, but instead becomes dynamical. In this picture the "boundary degrees of freedom" are nothing but the vibrational modes of this boundary surface.

We then determined the Hilbert space of the boundary states. In essentially all cases we found the number of states to be too few, or too many, to provide a microscopic source of black hole entropy.

In the cases for which the edge state phase space (43) is compact, we generically obtained the logarithm of the value expected from various thermodynamic considerations, cf. Eqs. (5) and (48). Here we note again that the logarithmic result (48) seems in qualitative agreement with the numerical results obtained by Srednicki [24], who determines the entanglement entropy for a massless scalar field in spacetimes of various dimensions. For $1+1$ dimensions, he gets an entropy proportional to $\ln R$, where, in his lattice calculation, $R$ was a radius midway between the outermost point traced over and the innermost point not traced over. However, the logarithmic result does not seem satisfy the simplest version of the first law of thermodynamics $T d S=d E$, which is obeyed by the Bekenstein-Hawking entropy.

On the other hand, if the phase space (43) (or the universal covering thereof) is not compact, there is no quantization condition on the thermodynamical entropy (or mass) and a finite degeneracy cannot be calculated by these methods.

In principle this infinity is less devastating than the previous result, where generically there were far too few quantum states. In the case of an infinite dimensional Hilbert space of the edge states one might still hope for some additional constraint that would restrict the number of physical states to a finite, but "large", number. One possibility, suggested to us by Carlip 40], is to look for a constraint that generates diffeomorphisms along the Killing direction. Such a constraint would be interpreted as a "remnant of the Wheeler-DeWitt constraint" that needs to be imposed on physical states. Unfortunately, a preliminary investigation suggests that there is no non-trival action of the generator of diffeomorphisms along the Killing vector on the edge degrees of freedom. The reasoning is as follows: As is obvious from (25), lines of constant $\Phi \sim X^{3}$ coincide with the Killing lines on the spacetime. So the generator $k$ of the diffeormorphisms into the Killing directions may not 
change $X^{3}$. Since, furthermore, on-shell $M=X^{a} X_{a}-\int{ }^{X^{3}} V(z) d z=$ const, according to (15) also $X^{a} X_{a}$, the only Lorentz invariant combination of $X^{a}$, is left invariant by $k$. Thus, up to possibly Lorentz transformations (in the frame bundle), $X^{i}$ will be left invariant by $k$. As a consequence also the local edge phase space variables $\widetilde{Y}^{2}$ and $\widetilde{Y}^{3}$ cannot be changed by $k$, cf. Eq. (40). As a result there is no non-trival action of $k$ on the edge degrees of freedom.

Actually at the Hamiltonian level it is easy to see that the appropriate generator in the phase space of the bulk action is nothing but the Casimir or mass functional $\widetilde{X}^{1} \sim M$. Thus the "dynamics" in the Killing direction resides precisely in the "abelian part" of the action, which was found to give a total divergence contribution to the boundary action only, cf. Eqs. (41, 42) and (18). This coincides also nicely with the fact that the Hamiltonian of our boundary action was found to vanish identically. From these considerations it appears that there is no need for a "remnant of the Wheeler-DeWitt constraint"; the symmetries in the Killing direction were already factored out at the classical level when part of the action was found to drop out as a total divergence.

Irrespective of these general considerations, the only candidate for such a generator that we can think of would be a function of only $Y^{3}$ (since $X^{3}=$ const are the Killing lines supposedly at least $Y^{3}$ should be left unchanged). But such an ansatz, considering $Y^{3}=$ const. as an additional constraint, does not seem to produce reasonable results either: In the example of the Lorentzian deSitter model, where the Poisson algebra (34) is linear, we may apply an algebraic quantization procedure analogous to the one used in the last subsection of the Appendix. We then have to count the eigenstates of the (hyperbolic) generator $Y^{3}$ in the $\operatorname{sl}(2, \mathbb{R})$-representations. In the principal continuous series this number is just two (two-fold degeneracy) while in the highest and lowest weight representations it is one only, cf., e.g., [41]. This attempt therefore also seems to fail.

It seems to us very important to be able to understand why the method of Carlip and Balachandran et al. appears to fail in the present context.

Possibly a further analysis of the "abelian part" of the action, $\int \widetilde{A}_{1} d \widetilde{X}^{1}$, that was found to yield only a total divergence contribution to the boundary action (42), could provide some insight. Dropping the rest of the action, this

\footnotetext{
${ }^{17}$ In any case, the generator $Y^{3}$ corresponds to (non-compact) frame bundle "rotations" (Lorentz transformations) and not to these diffeomorphisms, which act trivially on the edge degrees $Y^{i}$, as argued above.
} 
comes down to the study of an abelian $B F$-theory. For the latter the gauge theoretic approach applied in this paper did not yield any boundary modes, irrespective of the chosen boundary conditions. The Hamiltonian approach presented in the Introduction, on the other hand, suggests the existence of boundary modes, although their existence will depend decisively on the chosen boundary conditions $\left[\right.$ As discussed above, furthermore, $\widetilde{X}^{1} \sim M$ is the generator of the Killing time transformations on the Hamiltonian level. Last but not least, as remarked to us by Steve Carlip [40], if we regard the partition function of the boundary action (18) of Euclidean deSitter gravity without an imaginary unit in front of the action, then we may not drop the "total divergence" $\dot{\lambda}$, stemming again from the abelian part of the bulk; rather, maps $g(t)$ with a non-trivial winding number for lambda, will yield a contribution that goes as the exponential of $\sqrt{M}$. For these reasons we believe that if there is a remedy for the statistical mechanical entropy of the general class of $2 \mathrm{~d}$ models considered in the present paper, it should come from a different treatment of the abelian part of the action.

In view of the preceeding remarks on the conceptual difficulties in deriving the boundary action, on the other hand, it seems natural also to give up the idea that the boundary action is determined uniquely by the bulk action and the boundary conditions. Perhaps one should be more flexible about what boundary action to consider, a point of view that seems quite close in spirit to the considerations of some recent works of Balachandran et al. In the case of (四) it may be that some different boundary action than the one studied in the present paper, which may be postulated rather than derived, would yield a better agreement of $\ln N$ with the semiclassical result (5).

Last but not least we remark that in the treatment of the boundary action we did not take into account any interaction with the bulk degrees of freedom. In the language of Carlip the bulk variables entered as "external currents" only. In particular the mass $M$ entering in the boundary conditions was non-dynamical; one quantized the edge degrees on a background spacetime of fixed mass $M$. In this sense the present calculation was semiclassical only. It may also well be that a combined treatment of the coupled bulk-boundary

\footnotetext{
${ }^{18}$ In the context of, e.g., the Schwarzschild black hole the quantities $O_{\xi}$ stemming from the abelian part of the action are either to be identified with the mass $M$ and its conjugate "time"-variable $T$, found in the last two references of [20], or, for our boundary conditions, will be just quantities fixed on the boundary. Note also that, in the standard way of thinking about the Schwarzschild black hole, $M$ and $T$ are rather regarded as the bulk modes of the black hole.
} 
system would yield more reasonable results.

We close with a final word on the relationship between Carlip's calculation and the string theoretic calculations of the statistical mechanical entropy of black holes. Clearly neither method is completely successful: so far, Carlip's edge state method only seems to work for the 3-d BTZ (extremal and nonextremal) black holes; the stringy calculations yield the Bekenstein/Hawking entropy for certain 4 and higher dimensional extremal and near-extremal black holes. Furthermore, the stringy method appears to have relation with the spacetime geometry. Perhaps by understanding the relationship between the two methods for extremal 3-d black holes, we could understand the statistical mechanical origin of the higher dimensional, and more physically realistic, non-extremal black holes.

\section{Acknowledgement:}

The authors thank A. Barvinsky, S. Carlip, H.D. Conradi, D. Giulini, T. Jacobson, D. Marolf, and P. Schaller for useful discussions. T.S. is grateful also to the Erwin-Schroedinger Institute for hospitality during last summer. This work was supported in part by the Natural Sciences and Engineering Research Council of Canada as well as by the Austrian FWF project 10.221PHY. 


\section{Appendix: Counting States on the su(2)-coad- joint orbit}

In the following we present three different methods for quantizing the point particle action (17) with $g \in S U(2)$. The first of them is the most abstract one, but, in contrast to the successive two methods, it is this quantization procedure that is applicable in the more general context of quantizing a general action of the form (45).

In particular, in all cases where the phase space of the point particles governed by (45), i.e. the (connected components of the) level surface (43), is compact, the phase space is diffeomorphic to a two-sphere. Moreover, there then always exist spherical coordinates on the sphere such that the symplectic form $\Omega$ takes the form const. $(M) d(\cos \theta) \wedge d \varphi$. Correspondingly, Eq. (A.1) and everything derived from this thereafter is valid in these cases too. In particular the compact phase spaces are Kaehler and a holomorphic polarization yields $\operatorname{dim}$ (Hilbertspace) $N=n+1$. Only the relation between the integer $n$ and the mass $M$ will change from model to model, being determined by the Bohr-Sommerfeld condition Eq. (46).

\section{1) Geometric Quantization}

We will give a brief sketch of this method; for more details see, e.g., 34]. In the main text [following Eq. (19)] we identified the symplectic form as $\Omega=\sqrt{M} d(\cos \theta) \wedge d \varphi$, where $\theta$ and $\varphi$ are standard spherical coordinates on the two-sphere. In geometric quantization $\hbar^{-1} \Omega$ becomes the curvature form of a line bundle over the phase space, here $S^{2}$. Such a bundle is characterized by a winding number $n \in Z$, which may be thought of as the homotopy class of the map from the 'equator' $S^{1}$ to the structure group $C^{*} \sim U(1) \times \mathbb{R}_{+}$, and which coincides precisely with the Chern number $(1 / 2 \pi) \oint \hbar^{-1} \Omega$ of the bundle. This is the origin of (46), which lead to (20) for the case of (17).

The phase space $S^{2}$ is a Kaehler manifold. This is seen explicitly by introducing a new complex coordinate $z=\cot (\theta / 2) \exp (i \varphi)(\operatorname{Re}(z)$ and $\operatorname{Im}(z)$ are stereographic coordinates), leading to

$$
\hbar^{-1} \Omega=i n \frac{d z \wedge d \bar{z}}{(1+z \bar{z})^{2}}
$$

where we replaced $\sqrt{M}$ by $n \hbar / 2$ already, which allows for the (local) Kaehler potential

$$
K=n \ln (1+z \bar{z})
$$


where $\hbar^{-1} \Omega=i \partial \bar{\partial} K$.

The fastest way to determine the dimension of the Hilbert space is to apply a version of the Riemann-Roch theorem, cf., e.g., [42]: The number of independent holomorphic sections to a line bundle with Chern number $n \in \mathbb{N}_{0}$ over a two-sphere $S^{2}$ is $n+1$. This then equals the dimension of the Hilbert space and thus coincides with the degeneracy $N$.

The above argument can be made more explicit: In the patch of applicability of the variable $z$ we choose a canonical potential $\alpha:=-i \partial K$ $\left(\Rightarrow \hbar^{-1} \Omega=d \alpha\right)$, and the polarization vector $\partial_{\bar{z}}$. The physical wave functions are then seen to be holomorphic functions in this patch, for which we may take as a basis

$$
\Psi(z)=z^{k} \quad, k \in \mathbb{N}_{0} .
$$

A complementary patch is provided by introducing the complex variable $w=1 / z$ (stereographic coordinates with respect to the opposite pole). For reasons of symmetry $\Omega$ has the same form in these coordinates, (A.1) with $w \leftrightarrow z$. However, $K$ clearly changes in form and so does $\alpha$ :

$$
\alpha=-i n \partial \ln (1+w \bar{w})+d\left(\ln \left(w^{i n}\right)\right) .
$$

Such an $\alpha$ has neither a $\bar{z}$ component nor a $\bar{w}$ component; since moreover $\partial_{\bar{z}} \propto \partial_{\bar{w}}, \Psi$ is also holomorphic in the $w$ coordinates. To have $\Psi$ fit together into a global holomorphic section over all of the $S^{2}$, however, we learn from the second term in (A.4), which enforces an extra multiplicative gauge transformation $\exp \left(-i \ln w^{i n}\right)=w^{n}$ when switching the patches, that $\Psi(w)=w^{n-k}$. This is non-divergent at $w=0 \leftrightarrow z=\infty$ only if $k$ is further restricted:

$$
k=0,1,2, \ldots n .
$$

This again leads to $\operatorname{dim}($ Hilbert space $)=N=n+1$.

Two final remarks: First, the inner product between two states $\Psi_{1}$ and $\Psi_{2}$ in the $z$-chart turns out to be $\left\langle\Psi_{1} \mid \Psi_{2}\right\rangle \propto \int \overline{\Psi_{1}(z)} \Psi_{2}(z)\left[i d z \wedge d \bar{z} /(1+z \bar{z})^{n+2}\right]$. Second, in the above we did not take into account a metaplectic correction, cf. [34]. This may lead to a slightly corrected Bohr-Sommerfeld condition and, correspondingly, to a slight shift in the spectrum for $M$ (cf. also the following two subsections), which, however, is of little relevance for the present considerations.

\section{2) Elementary Oscillator Approach}


We now wish to quantize (17) by means of an elementary alternative method 23. For this purpose we first parametrize $g$ by

$$
g=\left(\begin{array}{cc}
z_{1} & z_{2} \\
-\bar{z}_{2} & \bar{z}_{1}
\end{array}\right)
$$

where

$$
z_{m}=\frac{1}{2}\left(\frac{q_{m}}{M^{1 / 4}}-i \frac{p_{m}}{M^{1 / 4}}\right) \quad, m=1,2,
$$

with the $p$ 's and $q$ 's real. In this parametrization, the action (17) takes the simple form

$$
S[q, p]=\oint_{E} d t\left(p_{1} \dot{q}_{1}+p_{2} \dot{q}_{2}\right)
$$

where we made use of the fact that $\left|z_{1}\right|^{2}+\left|z_{2}\right|^{2}=1$ due to $g \in S U(2)$. In terms of the phase space parameters $p_{m}, q_{m}$ this last restriction on the variables $z_{m}$ becomes a first class constraint, namely:

$$
\frac{1}{2}\left(\left(p_{1}\right)^{2}+\left(p_{2}\right)^{2}+\left(q_{1}\right)^{2}+\left(q_{2}\right)^{2}\right) \approx 2 \sqrt{M}
$$

This is of the form of the Hamiltonian of two coupled oscillators with frequency 1 and total energy $2 \sqrt{M}$. Moreover, the implementation of this constraint in a Dirac procedure becomes just the stationary Schroedinger equation for this auxiliary oscillator system. So here the standard result that the energy of oscillators becomes discrete on the quantum level yields the quantization condition (20) on $M$. Indeed $2 \sqrt{M}=\hbar\left(n_{1}+n_{2}+1\right), n_{m} \in \mathbb{I N}_{0}$ reproduces just this equation upon the substitution $n=n_{1}+n_{2}+1$. In this approach the degeneracy becomes $n$.

Note that in the previous, geometric approach the spectrum for $M$ had precisely the same form, except that $n \in \mathbb{N}_{0}$ while here $n \in \mathbb{N}$. Shifting $n \rightarrow n-1$ in the spectrum for $M$ of the previous subsection would yield complete agreement (including the degeneracy).

\section{3) Algebraic Approach}

From the general considerations in the paragraphs around Eqs. (43) and (45) it is clear that the quantization of the edge degrees of freedom of a general model described by a Lagrangian (田, 33) comes down to the quantization of the Poisson brackets

$$
\left\{Y^{i}, Y^{j}\right\}=\mathcal{P}^{i j}(Y)
$$


subject to the constraint (43):

$$
Y^{a} Y_{a}-\int^{Y^{3}} V(z) d z=M
$$

without which the bracket (A.10) would be not non-degenerate. In general the brackets (A.10) will be highly non-linear and an algebraic mechanism of implementing them as operator relations in some irreducible representation, respecting, furthermore, the constraint (A.11), will not be feasible. However, in the present case of the interest in the Appendix, (i.e. the edge dymanics of (the $S U(2)$-version of) (12)), the bracket (A.10) reduce to the linear $s u(2)$ brackets

$$
\left\{Y^{i}, Y^{j}\right\}=\varepsilon(i j k) Y^{k}
$$

with (A.11) becoming $(\vec{Y})^{2}=M$. The algebraic quantization of these brackets is well-known certainly. The irreducible representations are labelled by a half-integer valued spin $j$, they have dimension $2 j+1$, and the spectrum of the Casimir $(\vec{Y})^{2}$ is $j(j+1) \hbar^{2}$.

The variables $\vec{Y}$ may be found also directly starting from (17). It is a nice exercise to verify that, using (19),

$$
-2 \operatorname{tr}\left(\vec{T} g^{-1} T_{3} g\right)=\left(\begin{array}{c}
\sin \theta \cos \varphi \\
\sin \theta \sin \varphi \\
\cos \theta
\end{array}\right),
$$

where $\vec{T}$ may be represented by $-i \vec{\sigma} / 2$, the $\sigma_{i}$ being Pauli matrices. Performing the change of variables from $g$ to $\vec{Y}$ defined by $\vec{Y}:=-2 \sqrt{M} t r\left(\vec{T} g^{-1} T_{3} g\right)$, $(\vec{Y})^{2}=M$ is satisfied by construction, and the brackets A.10 follow immediately from the symplectic form $\Omega=\sqrt{M} d \cos \theta \wedge d \varphi$, obtained from (17) in the main text right after Eq. (19).

Let us finally compare the results of the present subsection to those of the preceding ones. For this purpose we first reformulate the present results in terms of the integer valued variable $2 j+1=: n$ :

$$
M=\frac{n^{2} \hbar^{2}}{4}-\frac{\hbar^{2}}{4} \quad, N=n \quad, n \in \mathbb{N} .
$$

In this form it is obvious that the difference between the algebraic approach and the previous oscillator approach resides merely in a shift of the 'zero point energy' by $-\hbar^{2} / 4$. Agreement with the geometric approach is obtained by 
the further shift $n \rightarrow n-1$ in the mass spectrum of subsection 1$)$. Moreover, we believe that a metaplectic correction to the geometric quantization will produce at least the first of these two shifts, possibly also the second one, which then would lead to complete agreement with (A.14).

\section{References}

[1] J.D. Bekenstein, Phys. Rev. D7, 2333 (1973); S.W. Hawking, Nature 248, 30 (1974).

[2] A. Strominger and C. Vafa, Phys. Letts. B379, 99 (1990).

[3] G. Horowitz, "The origin of black hole entropyin string theory", Proceedings of the Pacific Conference on Gravitation and Cosmology, Seoul, 1996 gr-qc/9604051; "Black hole microstates in string theory", in Matters of Gravity gr-qc/9609008.

[4] S. Carlip, Phys. Rev. D51, 632 (1995).

[5] S. Carlip, "The Statistical Mechanics of the Three-Dimensional Euclidean Black Holes", gr-qc/9606043 version 2 (1996).

[6] A.P. Balachandran, L. Chandar and A. Momen, "Edge States in Gravity and Black Hole Physics", gr-qc/9412019 (1994); "Edge States in Canonical Gravity", gr-qc/9506006 (1995).

[7] L. Susskind, L. Thorlacius and J. Uglum, Phys. Rev. D48, 3743 (1993).

[8] T. Jacobson, private communication.

[9] J.W. York, Phys. Rev. D33, 2092 (1986); J.D. Brown and J.W. York, Phys. Rev. D47, 1420 (1993); and references therein.

[10] M. Maggiore, Nucl. Phys. B429, 205 (1994).

[11] Cf., e.g., J. Fröhlich and U.M. Studer, Rev. Mod. Phys. 65, 733 (1993) and references therein.

[12] M. Banados, C. Teitelboim and J. Zanelli, Phys. Rev. Lett. 69, 1849 (1992); M. Banados, M. Henneaux, C. Teitelboim and J. Zanelli, Phys. Rev. D48, 1506 (1993). 
[13] A. Achucarro and P. Townsend, Phys. Lett. B180, 89 (1986); E. Witten, Nuc. Phys. B311, 46 (1988).

[14] E. Witten, Comm. Math. PHys. 137, 29 (1991).

[15] R. M. Wald, Phys. Rev. D48, R3427 (1993); V. Iyer and R. M. Wald, ibid, 50, 846 (1994).

[16] T. Banks and M. O'Loughlin, Nucl. Phys. B362, 649 (1991). See also R.B. Mann, Phys. Rev. D47, 4438 (1993) and S. Odintsov and I. Shapiro, Phys. Letts. B263, 183 (1991)

[17] D. Louis-Martinez, J. Gegenberg and G. Kunstatter, Phys. Letts. B321, 193 (1994).

[18] T. Fukuyama and K. Kamimura, Phys. Lett. B160, 259 (1985); K. Isler and C. Trugenberger, Phys. Rev. Lett. 63, 834 (1989); A. Chamseddine and D. Wyler, Phys. Lett. B228, 75 (1989); cf. also P. Schaller and T. Strobl, Phys. Lett. B337, 266 (1994).

[19] D. Cangemi and R. Jackiw, Phys. Rev. D50, 3913 (1994); D. Cangemi and R. Jackiw, Phys. Letts. B337, 271 (1994).

[20] B.K. Berger, D.M. Chitre, V.E. Moncrief and Y. Nutku, Phys. Rev. D5, 2467 (1973). W.G. Unruh, Phys. Rev. D14, 870 (1976); G.A. Vilkovisky and V.F. Frolov, Proc. 2nd Seminar on Quantum Gravity (Moscow 1981), ed. M.A. Markov and P.C. West (London: Plenum 1983), p267; P. Thomi, B. Isaak and P. Hajicek, Phys. Rev. D30, 1168 (1984); P. Hajicek, Phys. Rev. D30, 1178 (1984); J. Gegenberg and G. Kunstatter, Phys. Letts. B233, 331(1989); J. Gegenberg and G. Kunstatter, Phys. Rev. D47, R4192 (1993); T. Thiemann and H.A. Kastrup, Nucl. Phys. B399, 211 (1993); H.A. Kastrup and T. Thiemann, Nucl. Phys. B425, 665 (1994); K. Kuchar, Phys. Rev. D50 3961 (1994).

[21] D. Louis-Martinez, J. Gegenberg and G. Kunstatter, Phys. Rev. D51, 1781 (1995).

[22] A. Achucarro and M.E. Ortiz, Phys. Rev. D48, 1506 (1993).

[23] J. Gegenberg, G. Kunstatter and T. Strobl, "Statistical Mechanical Entropy of Two-Dimensional Black Holes", Proceedings of the 2nd Sakharov Symposium (to appear). gr-qc/9607055. 
[24] M. Srednicki, Phys. Rev. Lett., Vol. 71, 666 (1993).

[25] P. Schaller and T. Strobl, LNP 436, p. 98, Eds. A. Alekseev et al, Springer 1994 or gr-qc/9406027. Mod. Phys. Letts. A9 (1994), 3129. hep-th/9411163 or in the Proceedings of the International Workshop on 'Finite Dimensional Integrable Systems', p. 181-190, Eds. A.N. Sissakian and G.S. Pogosyan, Dubna 1995.

[26] P. Schaller and T. Strobl, Lecture Notes in Physics 469, p. 321, Eds. H. Grosse and L. Pittner, Springer 1996 or hep-th/9507020.

[27] T. Klösch and T. Strobl, Class. Quant. Grav. 13 (1996), 965.

[28] A. Sevrin and P. van Nieuwenhuizen, Phys. Rev. D50, 4157 (1994).

[29] N. Ikeda, Ann. Phys. 235, 435 (1994).

[30] S. Elitzur et al, Nucl. Phys. B326, 108 (1989); E. Witten, Comm. Math. Phys. 121, 351 (1989).

[31] S. Carlip and C. Teitelboim, Phys. Rev. D51, 622 (1995).

[32] B.M. Barbashov, V.V. Nesterenko and A.M. Chervjakov, Theor. Math. Phys. 40, 15 (1979); R. Jackiw in Quantum Theory of Gravity, ed. by S. Christensen, Adam Hilger, Bristol (1984); C. Teitelboim in Quantum Theory of Gravity, ed. by S. Christensen, Adam Hilger, Bristol (1984).

[33] B. Kostant, "Quantization and Unitary Representations", in Lectures in moder analysis III, ed. C.T. Taam, Lecture Notes in Mathematics, Vol 170, Springer, Berlin. (197); J.-M. Souriau, Structure des Systemes dynamiques', Dunod, Paris (1970).

[34] N.M.J. Woodhouse, Geometric Quantization, Oxford University Press (1980).

[35] J.E. Hetrick and Y. Hosotani, Phys. Letts. B230 88 (1989) and references therein. Cf. also S. Shabanov, Phys. Letts. B318 323 (1993).

[36] D. Louis-Martinez and G. Kunstatter, Phys. Rev. D52, 3494 (1995). 
[37] T. Klösch and T. Strobl, "Solutions of Arbitrary Topology and Kinks in 1+1 Gravity", hep-th/9607226.

[38] W. Kummer and S.R. Lau, Role of Quasilocal Energy and Boundary Conditions in the Canonical Formulation of all $1+1$ Models of Gravity, gr-qc/9612021, and references therein.

[39] A. Barvinsky and G. Kunstatter, "Exact Physical Black Holes States in Generic 2-D Dilaton Gravity", Phys. Letts. B (to appear), hepth/9606134.

[40] S. Carlip, Private communication.

[41] B.G. Wybourne, Classical groups for physicists, Wiley, New York, 1974. M. Natsuume and Y. Satoh, hep-th/9611041.

[42] M. Schlichenmaier, An Introduction to Riemann Surfaces, Algebraic Curves and Moduli Spaces, LNP 322, Springer, 1989. 